\title{
Malacoplaquia en un adolescente: a propósito de un caso
}

\section{Malacoplakia in a teenager: A case report}

\author{
Rubén Muñoz-Cedeño, ${ }^{{ }^{*}}$ (1) Gema Rodríguez-Chica, ${ }^{2}$ (]) Priscila Martínez-Ballesteros, ${ }^{3}$ (C) Viviana Paullan-Sani, ${ }^{4}$ (]) \\ Fabricio Vera-Zapata. ${ }^{5}$ (16)
}

\begin{abstract}
Gacceso abierTo
Citación:

Muñoz-Cedeño R, Rodríguez-Chica G,

Martínez-Ballesteros P, Paullan-Sani V, Vera-

Zapata F. Malacoplaquia en un adolescente:

a propósito de un caso. Rev Colomb

Gastroenterol. 2021;36(1):103-108. https://doi.

org/10.22516/25007440.475
\end{abstract}

Médico posgradista de Gastroenterología, Hospital de Especialidades Dr. Abel Gilbert Pontón, Universidad de Guayaquil, Ecuador.

2 Magister en Seguridad y Salud Ocupacional, Universidad Espíritu Santo. Médico residente de la unidad de cuidados intensivos maternos, Hospital Universitario, Universidad de Guayaquil. Guayaquil, Ecuador.

3 Médico especialista en Gastroenterología, Universidad de La Habana, Cuba. Coordinadora del posgrado de Gastroenterología, Universidad de Guayaquil. Formación en Ecoendoscopia en La Plata, Argentina. Líder del servicio de Gastroenterología del Hospital de Especialidades Dr. Abel Gilbert Pontón, Guayaquil, Ecuador.

4 Médico especialista en Gastroenterología, Universidad de La Habana, Cuba. Tutora del posgrado de Gastroenterología, Hospital de Especialidades Dr. Abel Gilbert Pontón; Universidad de Guayaquil, Ecuador.

5 Médico Gastroenterólogo, Hospital General de Guayaquil los Ceibos. Tutor del posgrado de Gastroenterología, Universidad de Guayaquil. Guayaquil, Ecuador.

*Correspondencia: Rubén Muñoz-Cedeño. drubenmunoz@gmail.com

Fecha recibido: $\quad 29 / 10 / 19$ Fecha aceptado: $08 / 01 / 20$

\section{Resumen}

La malacoplaquia es una enfermedad inflamatoria rara, granulomatosa y crónica, caracterizada por infiltrados agregados de histiocitos conocidos como inclusiones de Michaelis-Gutmann. El sistema urinario es el sitio afectado más común, seguido del tracto gastrointestinal. El pronóstico de la enfermedad depende de la extensión, la localización y la salud subyacente del paciente. Caso: se describe un caso clínico de un paciente masculino de 15 años de edad, sin antecedentes de salud de importancia, que inicialmente presenta la enfermedad a partir de los 7 años con hematoquecia y dolor abdominal de leve intensidad que mejoraba después de la defecación. Se realizó una colonoscopia en la que se visualizaron lesiones pseudopolipoideas en el colon sigmoide, las cuales fueron biopsiadas y reportaron pólipos inflamatorios con focos de agudización, numerosos histiocitos (cuerpos de Michaelis-Gutmann) y cambios reactivos del epitelio que diagnosticaron malacoplaquia. Conclusión: la malacoplaquia es una enfermedad muy rara en personas jóvenes sin enfermedad subyacente. Su prevalencia es desconocida, pero se han descrito más de 700 casos. Puede darse en todas las edades, con edad media al diagnóstico de 50 años. Los casos pediátricos son raros. Existe una mayor incidencia en hombres y no tiene predilección por la raza. El $60 \%$ a $80 \%$ de los casos afecta al tracto urinario (vejiga, riñones y uretra); el $15 \%$, al tracto digestivo (colon izquierdo, sigma, recto y estómago); y en menor porcentaje, otras zonas menos frecuentes como piel, pulmones y sistema nervioso central. El pronóstico de la enfermedad es generalmente bueno. Presentamos un caso de un joven sano de 15 años de edad sin enfermedad asociada evidente, que ilustra la rareza de esta presentación y la necesidad de un alto nivel de sospecha clínica para diagnosticar la enfermedad.

\section{Palabras clave}

Malacoplaquia, tracto gastrointestinal, colon.

\section{Abstract}

Malacoplakia is a rare, granulomatous, chronic inflammatory disease characterized by foamy histiocytic infiltrations known as Michaelis-Gutmann inclusions. The urinary system is the most commonly affected site, followed by the gastrointestinal tract. The prognosis of the disease depends on the extent, location, and underlying health status of the patient. This is the clinical case of a 15-year-old male patient with no significant health history. At age 7, the patient presented with hematochezia and mild abdominal pain that improved after defecation. A colonoscopy was performed, finding pseudopolypoid lesions in the sigmoid colon. Biopsy samples were taken, and the report showed inflammatory polyps with exacerbation foci, numerous histiocytes (Michaelis-Gutmann bodies), and reactive epithelial changes that lead to diagnose malacoplakia. Conclusion: Malacoplakia is a very rare disease in young people with no underlying disease. Its prevalence is unknown, but more than 700 cases have been reported. It can occur at all ages, with a mean age at diagnosis of 50 years. Pediatric cases are rare. There is a higher incidence in men, and it has no racial predilection. Between $60 \%$ and $80 \%$ of cases involve the urinary tract (bladder, kidneys, and urethra); $15 \%$ involve the digestive tract (left colon, sigmoid colon, rectum, and stomach); and, to a lesser extent, other areas such as skin, lungs, and central nervous system may be affected. The prognosis of the disease is usually good. This is the case of a healthy 15 -year-old male with no apparent associated disease that illustrates the rarity of this presentation and the need for a high level of clinical suspicion to diagnose the disease.

\section{Keywords}

Malacoplakia, Gastrointestinal tract, Colon. 


\section{INTRODUCCIÓN}

La malacoplaquia es una enfermedad inflamatoria rara, granulomatosa y crónica que afecta a muchos órganos como el tracto genitourinario, tracto gastrointestinal, piel, pulmones y sistema nervioso central (SNC) (1). Se encuentra con mayor frecuencia en el tracto genitourinario en un $60 \%$ a $80 \%$, y el segundo sitio más común es el tracto gastrointestinal, en un $15 \%$; y en menor frecuencia otras zonas como la piel, pulmones y SNC (2). Su prevalencia es desconocida, pero se han descrito más de 700 casos. Puede darse en todas las edades, con edad media de diagnóstico a los 50 años. Los casos pediátricos son raros. Tiene una mayor incidencia en hombres y no tiene predilección por la raza. Se asocia con otras afectaciones coexistentes como la sarcoidosis, tuberculosis, trasplante de órganos, afectaciones alérgicas, quimioterapia citotóxica, síndrome de inmunodeficiencia adquirida (SIDA), neoplasias, uso de esteroides y diabetes mal controlada $(3,4)$. Se diagnostica mediante histología con la presencia de grandes macrófagos de Hansemann, que contienen esférulas calcificadas laminadas conocidas como inclusiones de Michaelis-Gutmann (5, 6). El pronóstico de la enfermedad depende de la extensión, la localización y la salud subyacente del paciente (7).

\section{PRESENTACIÓN DEL CASO}

Se trata de un paciente de 15 años de edad, sin antecedentes patológicos previos, que fue remitido a nuestro hospital por hematoquecia. Sus antecedentes familiares son variados: madre diabética tipo 2, tía materna con esclerodermia, tía materna con amiloidosis y bisabuela con cáncer gástrico. Refirió el inicio de enfermedad a los 7 años de edad con cuadro clínico de hematoquecia y dolor abdominal de leve intensidad que mejoraba después de la defecación. En 2011 se realizó una colonoscopía con hallazgos de lesiones sobreelevadas pseudopolipoideas de 3 a 4 milímetros en el colon sigmoide y el recto con una biopsia que reportó malacoplaquia. Recibió tratamiento con ciprofloxacino $500 \mathrm{mg}$ vía oral por 14 días, que mejoró su sintomatología. En 2016 se realizó otra colonoscopia por presentar proctorragia y leve dolor abdominal. Nuevamente se describieron formaciones nodulares pseudopolipoideas en el sigmoides y el recto (Figura 1), con una biopsia que reportó pólipos inflamatorios con focos de agudización, numerosos histiocitos y cambios reactivos del epitelio. En este evento, recibió tratamiento con ciprofloxacino $500 \mathrm{mg}$ vía oral cada 12 horas por 14 días y presentó mejoría de la sintomatología. En julio de 2018, se realizó otra colonoscopia que informó lesiones pseudopolipoideas de distribución irregular con mucosa colónica normal, áreas planas de aspecto nacarado de bordes irregulares (Figura 2), con una biopsia que reportó pólipos inflamatorios, colitis crónica acentuada con tinción de ácido periódico Schiff (PAS), sin encontrar cuerpos de Michaelis-Gutmann.

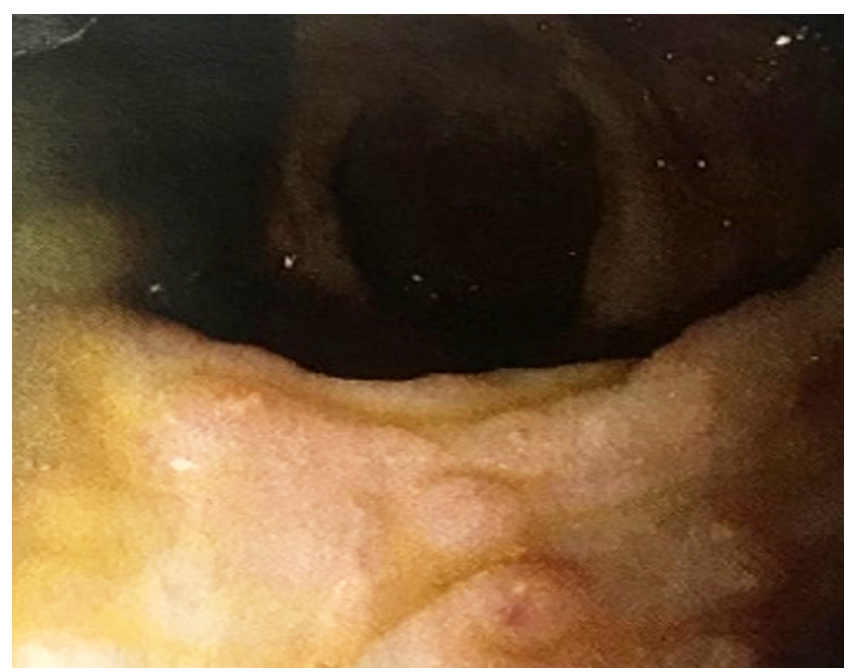

Figura 1. Videocolonoscopia en la que se observa la mucosa del colon sigmoide y recto con formaciones nodulares pseudopolipoideas.

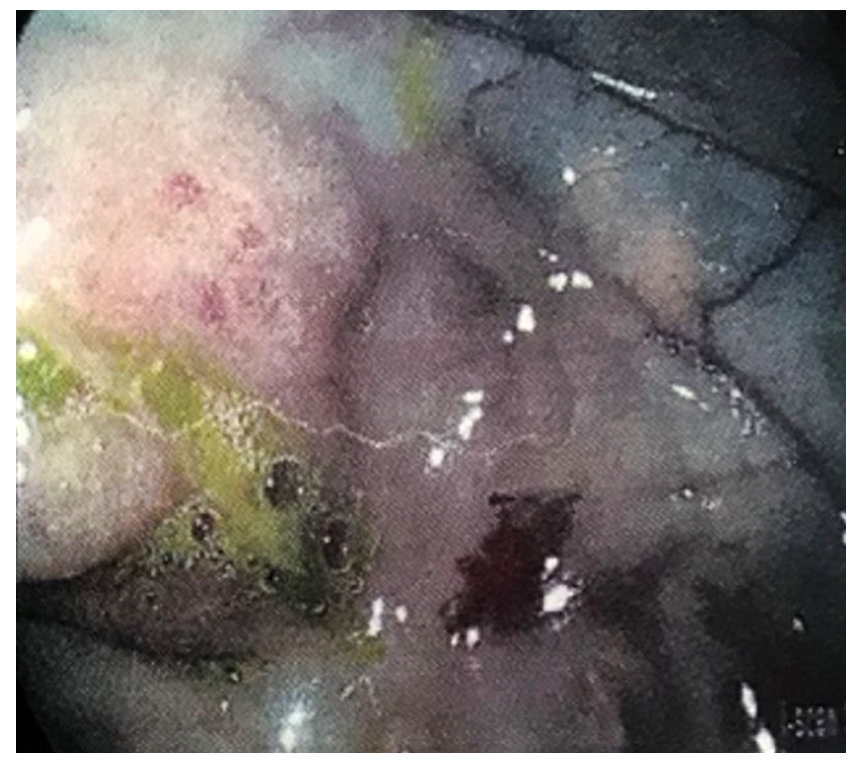

Figura 2. Lesiones pseudopolipoideas de distribución irregular con mucosa colónica normal, áreas planas de aspecto nacarado de bordes irregulares.

Ingresó a nuestro hospital por diarreas sanguinolentas de 4 a 5 veces en 24 horas, sin moco, ni pujo o tenesmo y leve dolor abdominal. Al examen físico presenta palidez mucocutánea, abdomen blando depresible con leve dolor en el marco colónico izquierdo. Sus signos vitales eran presión arterial de 110/70 $\mathrm{mm} \mathrm{Hg}$ y frecuencia cardíaca de 82 latidos por minuto. Se interrogó a la familiar, madre, quien refirió no conocer el diagnóstico de su hijo, a pesar 
de poseer informes endoscópicos e histopatológicos de malacoplaquia. Además, comentó que no seguía algún tipo de tratamiento, ni indicaciones dietéticas en casa. Se realizaron exámenes de laboratorio en los que se observa anemia, hipoalbuminemia, citomegalovirus (CMV), herpes virus reactivo inmunoglobulina $\mathrm{G}$ ( $\mathrm{IgG})$, calprotectina fecal disminuida y coproparasitario con Entamoeba coli y Entamoeba histolytica positivas. Se inició el tratamiento con ciprofloxacina y metronidazol intravenoso, y se plantea realizar el procedimiento endoscópico.

La información obtenida del paciente a través de las pruebas de laboratorio se presenta en la Tabla 1.

\section{Estudios endoscópicos y hallazgos histológicos}

En la videoendoscopia alta a nivel de la mucosa de antro se visualizó una lesión de 2 centímetros de diámetro de aspecto submucoso (Figura 3). En el duodeno se observaron lesiones pseudopolipoideas con exudado de fibrina en su superficie (Figura 4).

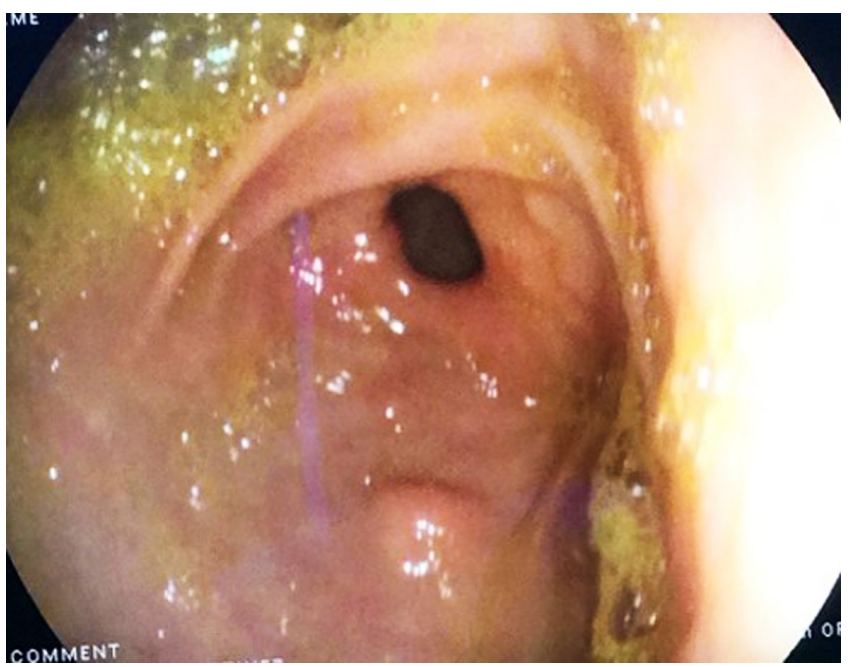

Figura 3. Lesión de $2 \mathrm{~cm}$ de diámetro de aspecto submucoso en la mucosa del antro.

La videocolonoscopia reveló a nivel del colon sigmoide y recto múltiples formaciones pseudopolipoideas sésiles, blandas, ulceradas y hemorrágicas, que medían de 5 a 15 mm de diámetro (Figuras 5 y 6 ).

El examen histológico reveló malacoplaquia, caracterizada por agregados de histiocitos granulares, de los cuales varios contenían cuerpos de Michaelis-Gutmann intracitoplasmáticos. Se confirmó histoquímicamente con tinciones periódicas de ácido peryódico de Schiff (PAS) (Figura 7).

Una vez iniciado el tratamiento con ciprofloxacino 200 mg intravenoso cada 12 horas, los síntomas de dolor abdominal y diarrea desaparecieron en una semana. Debido a
Tabla 1. Datos de laboratorio del paciente

\begin{tabular}{|c|c|}
\hline Prueba & Valor \\
\hline Leucocitos & 10300 \\
\hline Neutrófilos & $69,8 \%$ \\
\hline Linfocitos & $18,50 \%$ \\
\hline Monocitos & $8,60 \%$ \\
\hline Eosinófilos & $3 \%$ \\
\hline Basófilos & $0,10 \%$ \\
\hline Eritrocitos & 3,80 \\
\hline $\mathrm{Hb}$ & $9,60 \mathrm{~g} / \mathrm{dL}$ \\
\hline Hto & $30,20 \%$ \\
\hline VCM & $79 \mathrm{fL}$ \\
\hline Plaquetas & 260000 \\
\hline Albúmina & $2,6 \mathrm{~g} / \mathrm{dL}$ \\
\hline Urea & 23 \\
\hline Creatinina & 0,61 \\
\hline Bilirrubina total & $0,41 \mathrm{mg} / \mathrm{L}$ \\
\hline ALT & 15 \\
\hline AST & 19 \\
\hline TP & $16,1 \mathrm{~s}$ \\
\hline INR & 1,35 \\
\hline CMV IgG & Reactivo \\
\hline CMV IgM & No reactivo \\
\hline Epstein BAAR IgG & Negativo \\
\hline Epstein BAAR IgM & Negativo \\
\hline Herpes I y || IgG & Reactivo \\
\hline Ferritina & $241 \mathrm{ng} / \mathrm{mL}$ \\
\hline Vitamina $B_{12}$ & $268 \mathrm{pg} / \mathrm{mL}$ \\
\hline Hierro sérico & $19 \mu \mathrm{g} / \mathrm{dL}$ \\
\hline Ácido fólico & $13 \mathrm{ng} / \mathrm{mL}$ \\
\hline Transferrina & $166 \mathrm{mg} / \mathrm{dL}$ \\
\hline Calprotectina fecal & $116 \mu \mathrm{g} / \mathrm{dL}$ \\
\hline E. coli & Positivo \\
\hline E. histolytica & Positivo \\
\hline Coprocultivo & Negativo \\
\hline Hemocultivos aerobios y anaerobios & Negativo \\
\hline
\end{tabular}

ALT: alanina-aminotransferasa; AST: aspartato-aminotransferasa; Hb: hemoglobina; Hto: hematocrito; INR: índice internacional normalizado; TP: tiempo de protrombina; VCM: volumen corpuscular medio. 


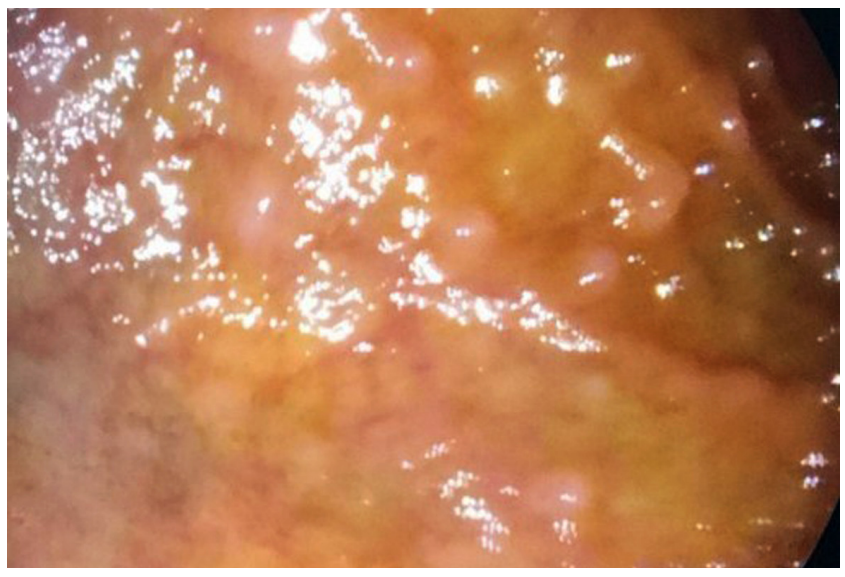

Figura 4. Lesiones pseudopolipoideas con exudado de fibrina en su superficie a nivel de la mucosa del duodeno observadas en la videoendoscopia alta.

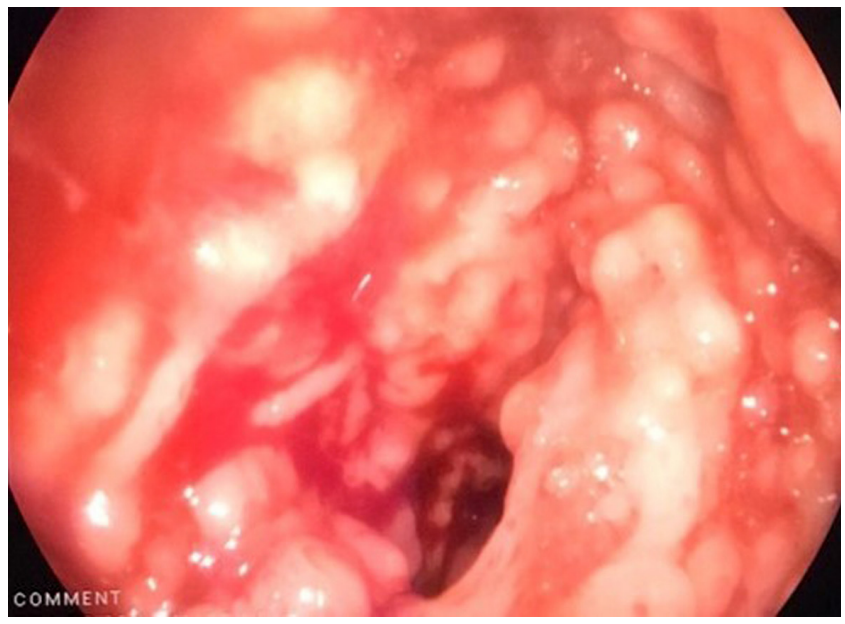

Figura 6. Videocolonoscopia a nivel de la mucosa del colon sigmoide y recto en la que se observaron múltiples formaciones pseudopolipoideas sésiles, blandas, ulceradas y hemorrágicas que midieron de 5 a $15 \mathrm{~mm}$ de diámetro.

esto, se dio alta médica con indicaciones de seguimiento por la consulta externa. A los 6 meses de control, el paciente se encontraba asintomático, con indicaciones nutricionales de consumo de ácido ascórbico y multivitamínicos, por lo que presentó ganancia de peso. Se informó a los padres del paciente las características de evolución, diagnóstico y pronóstico de la enfermedad, ya que el paciente tiene 7 años con el diagnóstico de malacoplaquia, pero los familiares desconocían de la misma. En las visitas de seguimiento ambulatorio han confirmado que actualmente está sano.

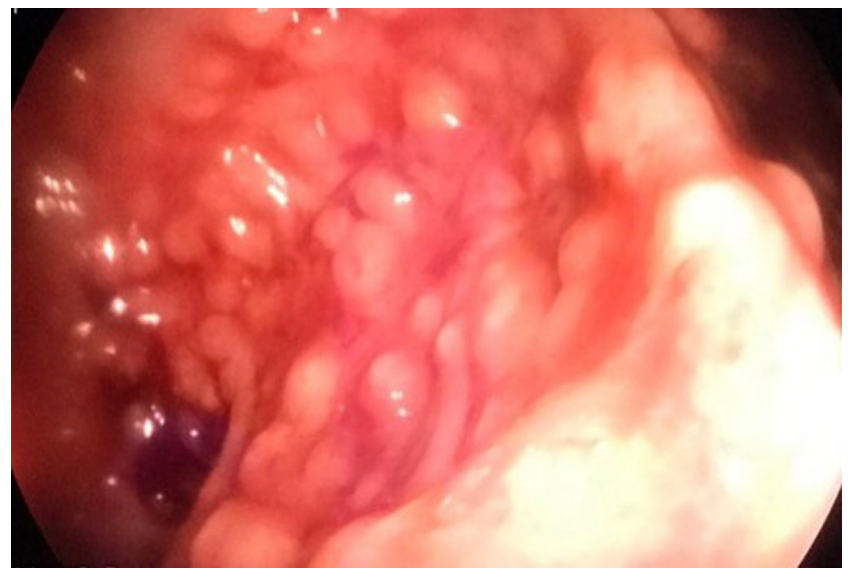

Figura 5. La mucosa del colon sigmoide mostró lesiones pseudopolipoideas pequeñas que se asemejan a una poliposis en la videocolonoscopia.

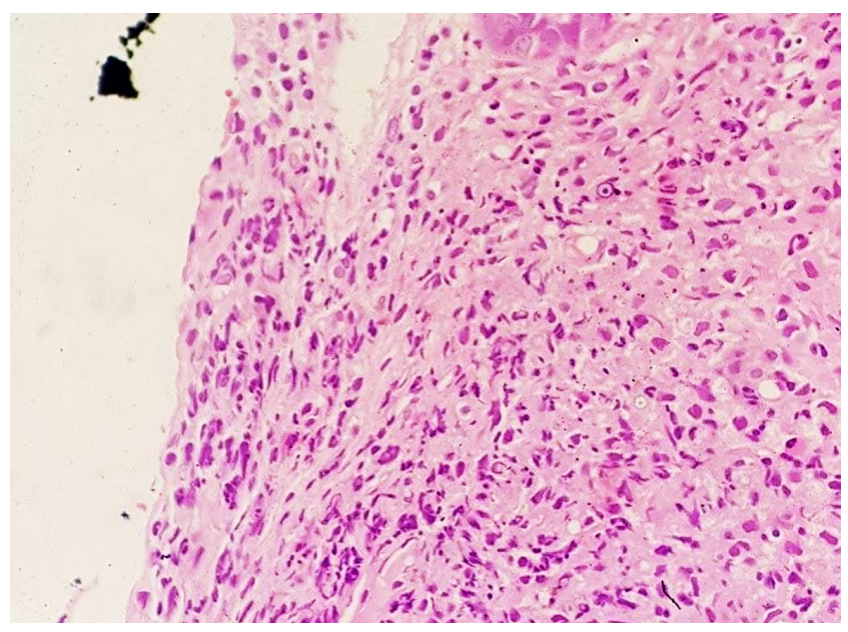

Figura 7. Hallazgo histológico que mostró inclusiones de agregados histiocitos y cuerpos de Michaelis-Gutmann con características patognomónicas de malacoplaquia.

\section{DISCUSIÓN}

La malacoplaquia es una enfermedad inflamatoria infrecuente, granulomatosa y crónica. Se diagnostica principalmente por rasgos característicos de histiocitos acumulados que contienen inclusiones citoplasmáticas, que son los cuerpos de Michaelis-Gutmann $(8,9)$. El segundo sitio que más comúnmente está afectado es el tracto gastrointestinal, con $11 \%$ de los casos, específicamente en el recto, seguido por el colon sigmoide $(10,11)$. La clínica es muy diversa, 
desde asintomática hasta manifestaciones como diarrea, dolor abdominal, sangrado rectal, obstrucción intestinal, vómitos, malestar general y fiebre $(12,13)$. La patogenia sigue siendo desconocida. Se han sugerido tres posibles mecanismos patogénicos: un organismo causal inusual, una respuesta inmune anormal o alterada y una respuesta anormal de macrófagos debido a una función lisosomal defectuosa (11). Se reportan casos de malacoplaquia con mayor frecuencia en pacientes con afectaciones coexistentes como trasplante de órganos, tuberculosis, terapia inmunosupresora, síndrome de inmunodeficiencia adquirida, neoplasias y diabetes mal controlada $(2,14)$.

La asociación de la malacoplaquia y adenocarcinoma de colon es rara. La mayoría de casos reportados por la literatura ocurre en el recto y colon sigmoide, de predominio en el sexo masculino; y en sí misma, la malacoplaquia no parece alterar el pronóstico del tumor, como se demostró en un metaanálisis de casos asociados con adenocarcinoma de colon $(6,15)$. En este paciente no está clara la etiología, por una parte, debido a que no tenía antecedentes médicos específicos ni era inmunodeprimido; y por otra parte, porque no tenía enfermedad maligna o crónica y no estaba en el grupo etario más afectado. Como ya se mencionó, este caso trata de un hombre adolescente, sano, sin enfermedades asociadas, con lesiones pseudopolipoideas. Se requirió realizar el diagnóstico diferencial con la enfermedad inflamatoria intestinal (EII) debido a la edad de presentación (16). En este caso, los síntomas del paciente mejoraron después de la administración del tratamiento antibiótico (ciprofloxacina por 14 días), puesto que se ha demostrado que la ciprofloxacina penetra bien los macrófagos y que, con dicho tratamiento, todas las lesiones granulomatosas desaparecen a largo plazo. Se puede curar la malacoplaquia con el tratamiento antibiótico correcto $(7,17)$.

Debido a la rareza de la condición, no hay pautas de tratamiento estándar. Sin embargo, actualmente los pilares del tratamiento son los antibióticos y el seguimiento con controles nutricionales asociados con multivitamínicos y ácido ascórbico, que han demostrado ser eficaces. Por este motivo, se debe informar a los familiares de las características de esta enfermedad, su evolución, diagnóstico y pronóstico, ya que es curable (18).

\section{CONCLUSIONES}

La malacoplaquia es una enfermedad muy rara en personas jóvenes sin enfermedad subyacente. Esto ilustra la rareza de este caso y la necesidad de un alto nivel de sospecha clínica para diagnosticar la enfermedad, cuya presentación es inespecífica. El pilar del tratamiento es la terapia con antibióticos.

\section{Conflictos de interés}

No existen conflictos de interés.

\section{Fuente de financiación}

Propia de los autores.

\section{REFERENCIAS}

1. Yared RA, Badran HA, Kamareddine MH, Ghosn Y, Bou Khalil R, El Ajamy K, Chouairy C, Farhat SG. Colonic Malakoplakia: A Rare Finding in a Healthy Male. Case Rep Gastroenterol. 2018;12(2):453-456. https://doi. org/10.1159/000492208

2. Yen JM, Soh NWY, Petersson F, Pandya G. Rectosigmoid malakoplakia. BMJ Case Rep. 2017;2017:bcr2017219464. https://doi.org/10.1136/bcr-2017-219464

3. Hyun KH, Shin HD, Kim DH. Malakoplakia in a healthy young female patient. Korean J Intern Med. 2013;28(4):475-80. https://doi.org/10.3904/ kjim.2013.28.4.475

4. Matter MJ, Gygi C, Gillet M, Gebhard S, Bouzourene $\mathrm{H}$. Malacoplakia simulating organ invasion in a rectosigmoid adenocarcinoma: report of a case. Dis Colon Rectum. 2001;44(9):1371-5. https://doi.org/10.1007/ BF02234800
5. Biggar WD, Crawford L, Cardella C, Bear RA, Gladman D, Reynolds WJ. Malakoplakia and immunosuppressive therapy. Reversal of clinical and leukocyte abnormalities after withdrawal of prednisone and azathioprine. Am J Pathol. 1985; 119(1):5-11.

6. Edmund L, Mohammed W. Colonic Carcinoma Associated with Malakoplakia. West Indian Med J. 2014;63(6):664-6. https://doi.org/10.7727/wimj.2014.079

7. Mitchell A, Dugas A. Malakoplakia of the colon following renal transplantation in a 73 year old woman: report of a case presenting as intestinal perforation. Diagn Pathol. 2019;14(1):22. https://doi.org/10.1186/s13000-0190799-z

8. McClure J. Malakoplakia of the gastrointestinal tract. Postgrad Med J. 1981;57(664):95-103. https://doi. org/10.1136/pgmj.57.664.95

9. Guha S, Liu H. Malakoplakia of the Pancreas with Simultaneous Colon Involvement: Case Report and Review 
of the Literature. Case Rep Pathol. 2015;2015:649136. https://doi.org/10.1155/2015/649136

10. Karasavvidou F, Potamianos SP, Barbanis S, Stathakis E, Psychos A, Kapsoritakis AN, Koukoulis G. Malakoplakia of the colon associated with colonic adenocarcinoma diagnosed in colonic biopsies. World J Gastroenterol. 2007;13(45):6109-11. https://doi.org/10.3748/wjg. v13.45.6109

11. Lewin KJ, Fair WR, Steigbigel RT, Winberg CD, Droller MJ. Clinical and laboratory studies into the pathogenesis of malacoplakia. J Clin Pathol. 1976;29(4):354-363. https:// doi.org/10.1136/jcp.29.4.354

12. Yousef GM, Naghibi B, Hamodat MM. Malakoplakia outside the urinary tract. Arch Pathol Lab Med. 2007;131(2):297-300. https://doi.org/10.1043/15432165(2007)131[297:MOTUT]2.0.CO;2

13. Alavian SM, Raissi Shabari F, Kamalian N. Malakoplakia of the colon: a case report and literature review, Med J Islamic Rep Iran. 2001;15(3):179-182.

14. Thorlacius H, Jerkeman A, Marginean FE, Toth E. Colorectal malakoplakia in a patient with hypogammag- lobulinemia. Gastrointest Endosc. 2018;88(3):563-565. https://doi.org/10.1016/j.gie.2018.04.001

15. Asiyanbola B, Camuto P, Mansourian V. Malakoplakia occurring in association with colon carcinoma. J Gastrointest Surg. 2006;10(5):657-61. https://doi. org/10.1016/j.gassur.2005.09.015

16. Jadhav MN, Patil RK, Kittur SK, Yadav J. Colorectal malakoplakia in a child presenting as multiple polyposis coli. Indian J Pathol Microbiol. 2015;58(1):86-8. https:/ / doi.org/10.4103/0377-4929.151197

17. van Furth R, van't Wout JW, Wertheimer PA, Zwartendijk J. Ciprofloxacin for treatment of malakoplakia. Lancet. 1992;339(8786):148-9. https://doi. org/10.1016/0140-6736(92)90212-1

18. Fudaba H, Ooba H, Abe T, Kamida T, Wakabayashi Y, Nagatomi H, Fujiki M. An adult case of cerebral malakoplakia successfully cured by treatment with antibiotics, bethanechol and ascorbic acid. J Neurol Sci. 2014;342(12):192-6. https://doi.org/10.1016/j.jns.2014.05.006 